\title{
USING FORMAL CONCEPT ANALYSIS TO EXPLORE HIDDEN KNOWLEDGE IN THE ASSESSMENT OF A MATH COURSE
}

\author{
Francisco Pérez-Gámez, Manuel Ojeda-Hernández, Ángel Mora Bonilla, \\ Domingo López-Rodríguez and Nicolas Madrid \\ Departamento de Matemática Aplicada - E.T.S.I.Informática - Universidad de Málaga, Spain*
}

\begin{abstract}
Since the emergence of COVID-19, online teaching and e-Learning has become essential in education. Actually, in at our University, we have had to move to a complete online teaching framework through the Moodle e-learning system. As a result, we have had to deploy new material as videos in Youtube channels, new exercises, tasks, live teaching, etc that have generated a huge amount of data that contains interesting information. In particular, we have used randomly generated exams from a bank of quizzes to evaluate the students. In this paper, we analyze the results of these quizzes using Formal Concept Analysis tools in order to check the hidden knowledge in the assessment process with the goal of improving the developed material for next years. In addition, we will analyze how the different exercises and tests relate to each other so that we can use this information in the following courses to improve our lectures.
\end{abstract}

\section{KEYWORDS}

e-Learning Assessment Tools, Formal Concept Analysis, Knowledge Mining, Concepts, Quizzes, Random Exams

\section{INTRODUCTION}

The term Blended Learning (Dziuban et al., 2018) refers to every teaching style that combines traditional face- to-face lectures with an online platform where some content is uploaded, namely theoretical material, quizzes, tasks or any other kind of stuff related to the module.

e-Learning has advantages such as allowing a self-paced learning for each one of the students, availability of the content at any time and community-based support, given in the form of forums where the students can ask and solve their own questions and their partners'. Usually we could say we approach our teaching using Blended Learning, that is, we provide on-site courses with live teaching but with strong support from online systems.

However, when our Blended Learning approach turns into a completely online course, as it has been mandatory with the situation caused by the COVID-19, some disadvantages might appear, such as a lack of focus, poorly motivated students can quickly fall behind in an online course; and the feeling of isolation the students can get, the impersonality, suppression of communication mechanisms such as body language, and elimination of peer- to-peer learning can make online learning a challenging experience for some students. This is a phenomenon we have experienced since class participation has decreased even though it had a specific weight in the marking scheme.

e-Learning is an interesting approach for learning that we can use to improve the quality of the teaching and learning process. Nowadays, in the framework of Spanish Universities, the use of Moodle is widely extended (Almansa-Martinez,2019) (Cabero-Almenara,2019). Moodle (Modular Object-Oriented Dynamic Learning Environment) is, at the moment, the most popular system for e-Learning purposes. In 2002, Martin Dougiamas presented the first version of Moodle based on socio-constructivist pedagogy providing a set of tools that support an inquiry- and discovery-based approach to online learning. Furthermore, Moodle allows for collaborative interaction among students as a standalone or in addition to conventional classroom instruction". 
The wide use of e-Learning platforms has brought about a huge amount of data that, with a proper processing, may bring out significant information very useful for teaching purposes. Classical techniques as Machine Learning or Data Mining can be used to extract knowledge from data. Currently, there are already many different approaches in the literature showing the advantages of using techniques of Machine Learning or Data Mining to e-Learning (Mohamad, 2013) (Romero, 2008), (Viloria, 2019). For example, it is possible to develop systems that adapt or recommend modifications of certain contents according to different students aspects defined by their behavior (Ashraf, 2020) (De Maio, 2012) (Asil Oztekin, 2013) (Hooshyar, 2020). Others applications of these kind of techniques to e-Learning are the development of tools to detect and prevent academic dropouts (Burgos, 2018), (Chung, 2019), (Chui, 2020), (Gray, 2019), the detection of problematic aspects in evaluation tasks (García 2011), the representation of feelings or preferences of students (Carmona, 2007), (Zengin, 2011) or the measurement of the student experience during an e-course (Shukor, 2015), (Hew, 2020) among others.

In our research we deal with the problem of searching for hidden patterns and relationships between different courses or lectures in university degrees. Specifically, in this paper we present some preliminary results obtained by using FCA techniques to the data collected from the course named EstMetNum (a module of Statistics and Numerical Methods) that has been taught completely in an e-Learning platform due to the COVID-19 restrictions. Data collected in this course include questions asked and debated by the alumni in the forums, quizzes answered, tasks proposed during the online lectures and knowledge bits proposed by the students in the glossaries, etc. This general problem of finding links between different lectures has been also addressed by other researchers, e.g., in (Yahya, 2019), (Buldu, 2010), but the difference with respect to our approach is that we use Formal Concept Analysis (FCA) (Ganter, 99), (Ganter, 2019) as the tool to extract the knowledge from the data, which although it has been applied in the context of e-Learning e.g., in (De Maio, 2012), to the best of our knowledge it has never been applied to this specific goal.

Formal Concept Analysis (FCA) is a solid mathematical framework to manage information, based on logic, lattice theory and Galois connections. It defines two explicit representations of the tacit knowledge present in a dataset, in the form of concepts (closed sets under a closure operator, that is, entities characterized by the non-formalized relationships among the attributes or features in a dataset) and implications, which can be seen as exact association rules.

The mechanisms used to extract concepts from a dataset in FCA allow us to hierarchically organize them in the so-called concept lattice. An important aspect to emphasize is that the concept lattice captures all the implicit knowledge that can be deduced from a formal context.

FCA provides the methods to find those representations, more precisely to extract the concepts and implications that can be deduced from the dataset, and introduces a logic to reason and infer new knowledge. In this sense, FCA allows to discover knowledge in datasets analogously to what other techniques (e.g. in Machine Learning) do, but this logic-based approach is more suitable to provide explainable answers when dealing with real-world datasets. Comparing our approach with other techniques used in machine learning and data mining such as association rules, FCA extracts more knowledge, since it gives not only the implications (exact association rules) but also the concepts organized in a hierarchical structure, the concept lattice. Moreover, our team has developed a logic to manipulate implications and to build automated methods to reason with them.

In this work, we approach using FCA the retrieval of the concepts from a dataset with the marks obtained in the different exercises and test using Moodle quizzes. In this way, Section 2 shows the material and methods we have developed in our online course explaining the generation of the quizzes using $\mathrm{R}$ language to build a huge bank of quizzes and how we have approached the use of FCA to extract knowledge. The results obtained are explored in Section 3 and some conclusions and future works appear in the last section.

\section{MATERIALS AND METHODS}

We analyze in this work the results (marks) obtained from a set of quizzes extracted from Moodle concerning the subject Statistics and Numerical Methods in the Bachelor degree of Telecommunication Systems Engineering and in the Bachelor degree of Electronic Systems Engineering. Of course, the data has been anonymized to protect the personal information of the students. 
Although we have used extensively the habitual tools that Moodle provides, and due to a big number of students (70 in each course), we have decided to generate a large question bank by means of $\mathrm{R}$ language and the R/exams package (http://www.r-exams.org). This open-source package exams, for the R language, provides a one-for-all approach to automatic exams generation. We develop dynamic exercise models with random parameters to build patterns of exercises and generate random exams. The questions were exported to Moodle using the exams2moodle function of the R/exams package. We could ensure that different students tackled different tests from a question bank of about 5,000 questions.

Moreover, during the course, some similar exercises generated in the same way were solved by students using a self-assessment question bank. The traditional class in the classroom has been moved to online teaching, then we reinforced the existent material in the Moodle course with new learning units: lectures using videos via a private Youtube channel together with online live lessons using Google Meet and/or Microsoft Teams, quizzes, tasks, forums, available material online, etc.

Although we believe that the work with all this material has been very satisfactory, we need to objectively measure the students' learning processes in order to give the right marks for each student's work. And the random exams generated from $\mathrm{R}$ language have been the main tool for assigning the final grade/mark.

The goal in this works is on the one hand to translate the intense work done in this hard situation caused by COVID and on the other hand to communicate the results we have achieved in the course.

The csv file with the marks of the different exercise patterns has the following structure:

- Three main statistics exams and three main numerical methods exams: Descriptive Probability Test (StD in the dataset), Random Variables Test (StR) and Matlab Statistical Test (StM), Non Linear Equations Test (NtNle), Numerical Nonlinear Equations and Solving Linear Systems Test (NtNleLs), Numerical Interpolation-Approximation-Derivation-Integration Test (NtIaDi) and Numerical Matlab Test (NtM).

- Two specific exercises from statistics and five specific exercises from numerical methods: Regression Exercise (Ser), Variables Distributions ( $\mathrm{SeV}$ ), Numerical Nonlinear Equations Exercise (NeNle), Numerical Interpolation Exercise (NeI), Numerical Non Classical Interpolation (NeNci), Numerical Exercise Approximation (NeA), Numerical Matlab Exercise (NeM).

- The final mark of the students (FinalMark).

As a summary of the goal of the paper, can we extract interesting knowledge from the marks in the exercise patterns? Some questions are raised as motivation of the analysis of the results we introduce in the rest of the paper using Formal Concept Analysis, a mathematical tool to discover knowledge:

- Which exercise patterns have been more decisive in the final marks?

- Is there any hard exercise that students have not approached adequately?

- Have the specific exercises been important in the final mark?

- Is there any interaction among some categories of the specific exercises or between specific exercises and main exams?

The data to be analyzed for FCA is a binary relation between a set of objects and a set of attributes. In a first stage of the analysis we will use classical FCA then we binarize the csv considering 1 if the student passes this exam or exercise. Next figure shows the binary relation named fc_en from the the csv file (en_bin.csv): 


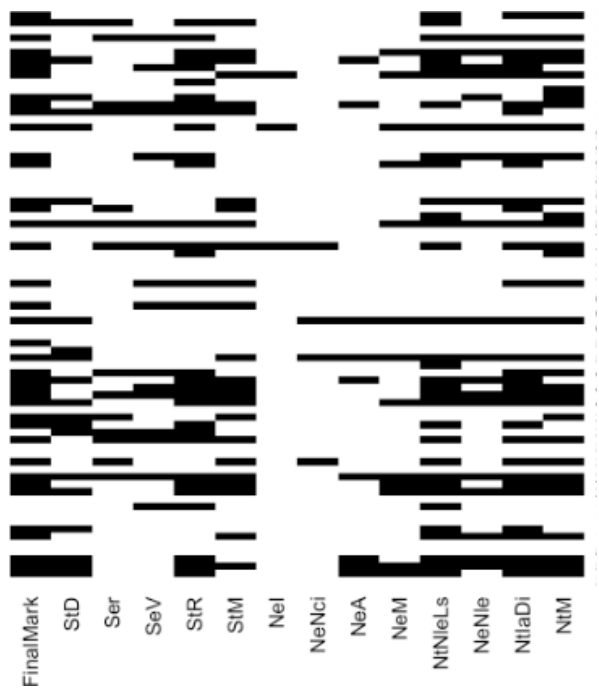

Figure 1. Binary relation

Due to the limitations of space, in this work we do not introduce an extensive summary of Formal Concept Analysis. See (Ganter, 99), (Ganter, 2019) for more details on FCA.

The binary table is named in FCA the formal context. It is a triple $\boldsymbol{K}=\preccurlyeq G, M, I>$ where $G$ is a set of objects and $M$ a set of attributes and $I \subseteq G \times M$ is a binary relation. $\preccurlyeq g, m>\in I$ means that the object $g$ has the attribute $m$. Two mappings $\uparrow: 2^{G} \rightarrow 2^{M}$ and $\downarrow: 2^{M} \rightarrow 2^{G}$ named concept-forming operators, are defined as follows: for any $X \subseteq G$ and $Y \subseteq M$,

$$
\begin{gathered}
X^{\uparrow}=\{m \in M \mid \preccurlyeq g, m>\in I, \text { for all } g \in X\} \\
Y^{\downarrow}=\{g \in G \mid \preccurlyeq g, m>\in I, \text { for all } m \in Y\}
\end{gathered}
$$

The meaning of $X \uparrow$ is the subset of all attributes shared by all the objects in $X$ and $Y \downarrow$ is the subset of all objects that have the attributes in $Y$. Without going into details, in FCA it has been proved that these mappings constitute a Galois connection, therefore, both compositions are closure operators and it is the key point to find the fixed points in which a set of object shares a set of attributes and this set of attributes shares the same set of objects, that is, a concept.

A pair of subsets $\preccurlyeq X, Y>$ with $X \subseteq G$ and $Y \subseteq M$ such that $X \uparrow=Y$ and $Y \downarrow=X$ is called a formal concept. is named the extent and $Y$ the intent of the concept. The formal concepts have an order relation, and the set of all the formal concepts, denoted by $(\boldsymbol{G}, \boldsymbol{M}, \boldsymbol{I})$, is a lattice, named concept lattice.

We have used R language to develop the analysis of the quizzes using FCA, and specifically the R package named fcaR(https://CRAN.R-project.org/package $=$ fcaR). This package allows to extract the knowledge inside a binary dataset using FCA (See https://github.com/neuroimaginador/fcaR for some vignettes explaining the use of this package).

Using fcaR the user can extract in a easy way the concepts, the full concept lattice, relationships between attributes, etc. In the following section, we show how we can use fcaR to extract the hidden knowledge in the datasets with the quizzes marks.

\section{RESULTS}

To answer the questions that we have presented in the previous section, we have explored the dataset using FCA methods. First, from the original binary dataset, we have built its concept lattice to explore the set of items (topics, exercises, tests) which share a common behaviour among students.

Since we are interested in frequent behaviours, in many cases we have to set a threshold on the support of the concepts we are studying. The support of a set is the proportion of cases in which the set appears in the dataset, among all cases. Depending on the variability of the data, the support threshold is also variable and will depend on the number of concepts that can be considered significative. 
This first approach has given a concept lattice composed by 187 concepts. With such a high amount, the lattice plot (called Hasse diagram) does not fit in standard paper sizes, thus we present only the lattice for concepts whose support exceeds $42 \%$ (that is, concepts appearing in at least $42 \%$ of the cases in the dataset) in the next figure:

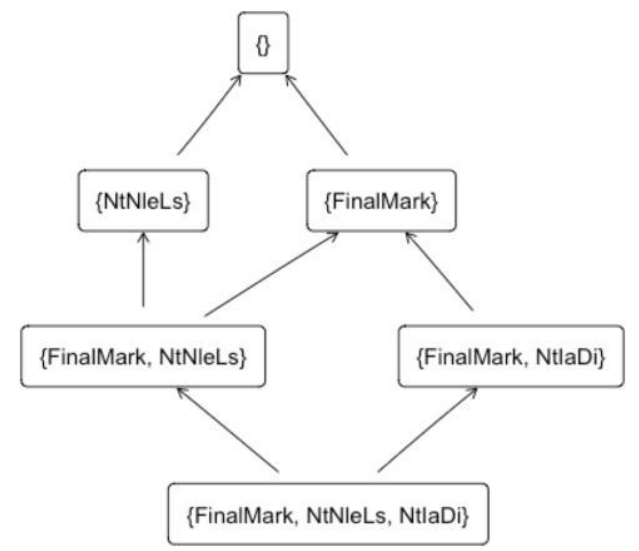

Figure 2. Concepts appearing in at least $42 \%$ of the cases in the dataset

We'll look at the so-called irreducible elements, the minimal elements needed to rebuild the whole lattice. They provide essential information about the frequent co-occurrences inside the dataset. This irreducible elements are those with only an upper-neighbour in our plot, so they are $\{$ NtNleLs\}, isep $\{$ FinalMark\}, istep $\{$ FinalMark, NtIaDi\}. Among those, only two of the concepts contain information about the FinalMark item. The first one,

\{FinalMark\}, means that essentially, passing the subject can be done in multiple ways and there is no a common pattern of items that co-occur that implies passing the subject. But the other concept, \{FinalMark, $\mathrm{NtIaDi}\}$, means that there is one fundamental test in the subject (the one about interpolation, approximation, differentiation and integration) that is key in the sense that it usually leads to the obtention of a good final mark.

This first analysis answers the questions "Which exercise patterns have been more decisive in the final marks?" and "Have the specific exercises been important in the final mark?". We expand this analysis to approach the remaining open questions.

Let us now consider two independent contexts, one for students who passed the subject and another for the students who did not. Again, the actual number of concepts in the concept lattice is high, so we must impose a threshold to only visualize the most frequent patterns, as in the next figure:
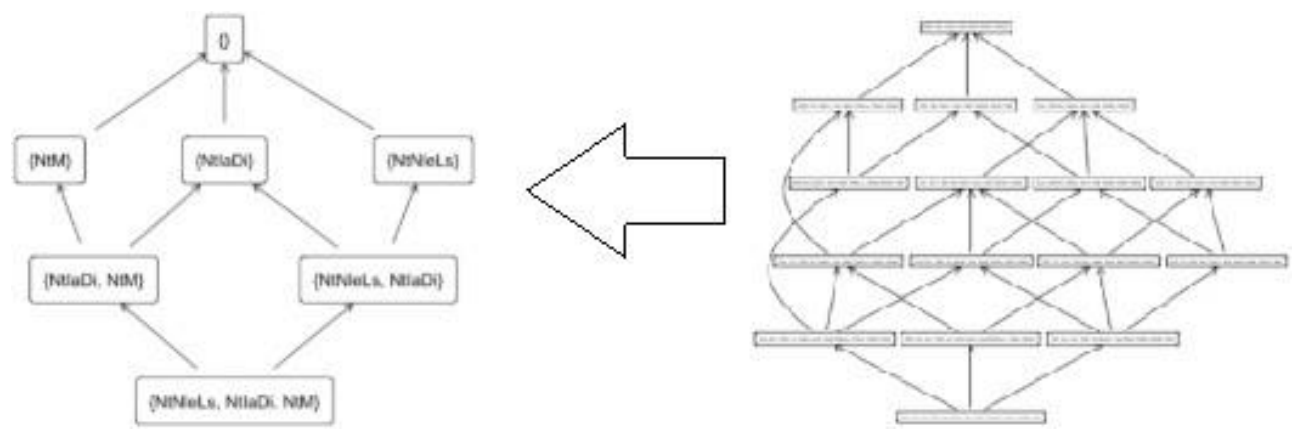

Figure 3. Left sublattice (figure 2) and right complete lattice

We can see that even imposing only frequent concepts, in the second lattice, concepts are difficult to read. This is due to the fact that concepts in that lattice contains many attributes, that is, many items (topics, tests, etc.). To have a better readability of those lattices, as before, we study the irreducible elements. 
In the first case, for students which passed, the most important concepts, defining what items clearly suggest the student is performing well in the subject, are $\{\mathrm{NtM}\},\{\mathrm{NtIaDi}\}$ and $\{\mathrm{NtNleLs}\}$. That is, we confirm that $\mathrm{NtIaDi}$ (the test on interpolation, approximation, differentiation and integration) is very important in the subject and thus passing it is a strong attribute of students who pass the subject. This analysis also allows us to identify the another two items (the MATLAB exam and the test on the resolution of numerical equations and systems) that are important in passing the subject.

For students who didn't pass, we study their fails, that is, in which items they failed that conducted to failing the subject. If we explore the irreducible elements of the lattice, \{StM, NeI, NeNci, NeA, NeM, NeNle, NtIaDi, NtM $\},\{$ StM, NeI, NeNci, NeA, NeM, NtNleLs, NeNle, NtIaDi \}, \{Ser, StM, NeI, NeNci, NeA, NeM, NeNle, NtIaDi $\},\{$ Ser, SeV, StM, NeI, NeNci, NeA, NeM, NeNle, NtIaDi $\}$ and $\{$ StD, Ser, StM, NeI, NeNci, NeA, $\mathrm{NeM}, \mathrm{NeNle}, \mathrm{NtIaDi}$, we find that these are items that students frequently fail where they fail the subject: the students fail a lot of tests; there is not a single item which if they fail then they fail the subject. Thus, failing the subject has not a single reason inside the subject. It can be hypothesized that the reason can be a lack of previous mathematical knowledge and skills in part of the students.

Thus, with this analysis, we have given response to "Which exercise patterns have been more decisive in the final marks?", "Is there any hard exercise that students have not approached adequately?", and "Have the specific exercises been important in the final mark?".

The last question to answer is related to the relationship between items in the subject, that is, if there is any set of items, tests, exercises, etc., whose behaviour among students is the same. This is related to the notion of statistical correlation. We build in this case a formal context by binarizing the correlation between items in the original formal context. We can then build the lattice of frequent correlated items in the subject, which we present in the next figure:

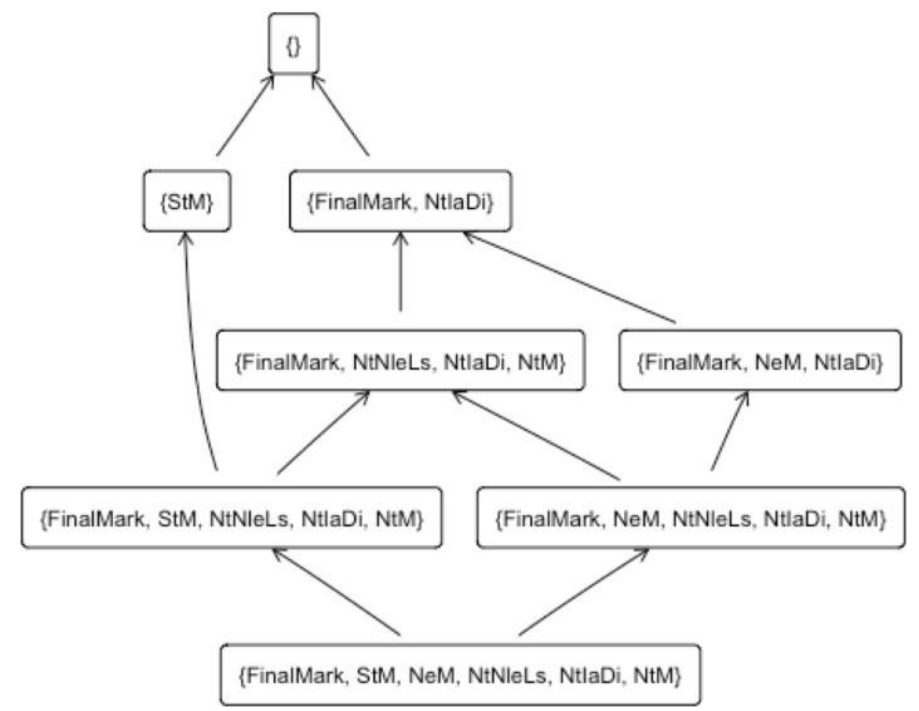

Figure 4. Lattice of frequent correlated items

The irreducible concepts in this lattice with more than one element, \{FinalMark, NtIaDi\}, \{FinalMark, NtNleLs, NtIaDi, NtM\} and \{FinalMark, NeM, NtIaDi \}, indicate which areas share the same behaviour among most students, that is, in every irreducible concept, all items are highly related, meaning that most students pass all of the corresponding tests or most students fail all of them. It is remarkable that in the three concepts the item NtIaDi appears accompanying the FinalMark, corroborating our previous findings: one of the key tests in the subject is the one for numerical interpolation, approximation, differentiation and integration. 


\section{CONCLUSIONS AND FUTURE WORK}

We have presented our experience on how to evaluate a full online course in our university due to the Covid restrictions. It was necessary to implement an online assessment and the use of quizzes was the best option. A huge quiz bank has been developed using $\mathrm{R}$ language and the R/exams package. A random exam for each student has been done and we have evaluated the students with all the marks obtained.

We have used in this work FCA to extract knowledge from the dataset with the marks of students in the quizzes. Some interesting patterns arise from this knowledge obtaining in our analysis the important topics, exercises and tests and the relationship among them. We show the concept lattice obtained and how it is possible to use FCA to reveal significant gaps in the evaluation process. We have obtained interesting patterns concerning the assessment units used in this course: some units appear frequently for the students passing the course (numerical interpolation, approximation, differentiation and integration); some units are detected as key tests; there is no test significative enough to make a student automatically fail (when the student fails, more than one test is failed); the subject can be succeeded in several ways and there is not a common pattern of items that co-occur when the students pass the subject. In general, the knowledge appearing in the concept lattice could reveal gaps, hard tests for students, unique ways to pass the subject doing the same test, etc.

This is the first step in our study, our short-term goals we want to extend our study using fuzzy formal concept analysis, where we can grade the marks and to obtain a deeper knowledge. Also, we are going to make changes in our lectures taking the results in this study into account and we will repeat this study in order to check if we have improved or new changes could be suggested.

\section{ACKNOWLEDGMENT}

This work has been partially supported by the project TIN2017-89023-P of the Science and Innovation Ministry of Spain, co-funded by the European Regional Development Fund (ERDF), UMA18-FEDERJA-001 and PIE15-130.

\section{REFERENCES}

Almansa-Martínez,et al,2019, Functionalities of moodle and edmodo in the middle and higher educations. In Revista de Comunicación de la SEECI, (Vol 50, 87-105).

Asil Oztekin, et al, 2013. A machine learning-based usability evaluation method for e-Learning systems. In Decision Support Systems (Vol 56, pp 63-73)

Ashraf, M. et al, 2020. An Intelligent Prediction System for Educational Data Mining Based on Ensemble and Filtering approaches. In Procedia Computer Science (Vol. 167, pp. 1471-1483). Elsevier B.V.

Buldu, A. and Üçgün, K, 2010. Data mining application on students' data. In Procedia - Social and Behavioral Sciences (Vol. 2, pp. 5251-5259). Elsevier.

Burgos, C. et al, 2018. Data mining for modeling students' performance: A tutoring action plan to prevent academic dropout. Computers and Electrical Engineering, 66, 541-556.

Cabero-Almenara, et al, 2019. Technical and Didactic Knowledge of the Moodle LMS in Higher Education. Beyond Functional Use. Journal of New Approaches in Educational Research, (Vol 8, 25-33)

Carmona, C., et al, 2007. Discovering Student Preferences in e-Learning.

Chui, K. T. et al, 2020. Predicting at-risk university students in a virtual learning environment via a machine learning algorithm. Computers in Human Behavior, 107, 105584.

Chung, J. Y. and Lee, S., 2019. Dropout early warning systems for high school students using machine learning. Children and Youth Services Review, 96, 346-353.

De Maio, C. et al, 2012. RSS-based e-Learning recommendations exploiting fuzzy FCA for Knowledge Modeling. Applied Soft Computing Journal, 12(1), 113-124.

Dziuban, C. et al, 2018. Blended learning: the new normal and emerging technologies. International Journal of Technology in Higher Education. 15(3)..

Ganter, B. and Wille, R. (1999). Formal Concept Analysis: Mathematical Foundations. Springer. Ganter, B. and Obiedkov, S. (2016). Conceptual Exploration. Conceptual Exploration, 1-315. 
García, E. et al, 2011. A collaborative educational association rule mining tool. Internet and Higher Education, 14(2), $77-88$.

Gray, C. C. and Perkins, D., 2019. Utilizing early engagement and machine learning to predict student outcomes. Computers and Education, 131, 22-32.

Hew, K. F. et al, 2020. What predicts student satisfaction with MOOCs: A gradient boosting trees supervised machine learning and sentiment analysis approach. Computers and Education, 145, 103724.

Hooshyar, D. et al,, 2020. Open learner models in supporting self-regulated learning in higher education: A systematic literature review. Computers and Education, 154, 103878.

Mohamad, S. K. and Tasir, Z., 2013. Educational Data Mining: A Review. Procedia - Social and Behavioral Sciences, 97, 320-324.

Romero, C., et al, 2008. Data mining in course management systems: Moodle case study and tutorial. Computers and Education, 51(1), 368-384.

Shukor, N. A., et al., 2015. An Examination of Online Learning Effectiveness Using Data Mining. Procedia - Social and Behavioral Sciences, 172, 555-562.

Viloria, A. et al, 2019. Determinating student interactions in a virtual learning environment using data mining. In Procedia Computer Science (Vol. 155, pp. 587-592). Elsevier B.V.

Waheed, H. et al, 2020. Predicting academic performance of students from VLE big data using deep learning models.

Computers in Human Behavior, 104.

Yahya, A. A. and Osman, A., 2019. Using Data Mining Techniques to Guide Academic Programs Design and Assessment. In Procedia Computer Science (Vol. 163, pp. 472-481). Elsevier B.V.

Zengin, K., Esgi et al, 2011. A sample study on applying data mining research techniques in educational science: Developing a more meaning of data. In Procedia - Social and Behavioral Sciences (Vol. 15, pp. 4028-4032). Elsevier. 